\title{
Hirudotherapy for Neonatal Limb Ischemia During ECMO Support: A Word of Caution
}

\author{
Joseph Resch ${ }^{1}$, Sameh Said ${ }^{2}$, Marie Steiner ${ }^{1}$, and Arif Somani ${ }^{1}$ \\ ${ }^{1}$ University of Minnesota Medical Center \\ ${ }^{2}$ University of Minnesota System
}

February 7, 2021

\begin{abstract}
Introduction: Disordered coagulation, clot formation and distal limb ischemia are complications of extracorporeal membrane oxygenation (ECMO) with significant morbidity and mortality. Medicinal leech therapy (hirudotherapy) has been attempted in plastic and orthopedic surgeries to improve venous congestion and salvage ischemic flaps. To our knowledge this has not been reported in pediatric cardiac surgery or during ECMO support. We present a complex neonate whose ECMO course was complicated by distal limb ischemia for whom leech therapy was attempted. Patient and Intervention: A 2 week-old $2.7 \mathrm{~kg}$ infant required ECMO support secondary to perioperative multiorgan system dysfunction following repair of critical coarctation and ventricular septal defect. Despite systemic anticoagulation, his clinical course was complicated by arterial thrombus, vasopressor-induced vascular spasm and bilateral distal limb ischemia. Medicinal leech therapy was tried after initially failing conventional measures. Result: Following the third leech application, this patient developed significant hemorrhage from the web space adjacent to the left great toe. An estimated $450 \mathrm{~mL}$ of blood loss occurred and more than $300 \mathrm{~mL}$ of blood product transfusions were required. He ultimately progressed to irreversible systemic end organ dysfunction and comfort care was provided. Conclusion: The use of medicinal leech therapy in pediatric cardiac surgery may be considered to minimize the consequences of advanced limb ischemia and venous congestion. However, this should be used with caution while patients are systemically anticoagulated during ECMO support. A directed review is presented here to assist in determining optimal application and potential course of therapy.
\end{abstract}

\section{Hosted file}

Hirudotherapy Manuscript.DOCX available at https://authorea.com/users/394288/articles/507699hirudotherapy-for-neonatal-limb-ischemia-during-ecmo-support-a-word-of-caution 


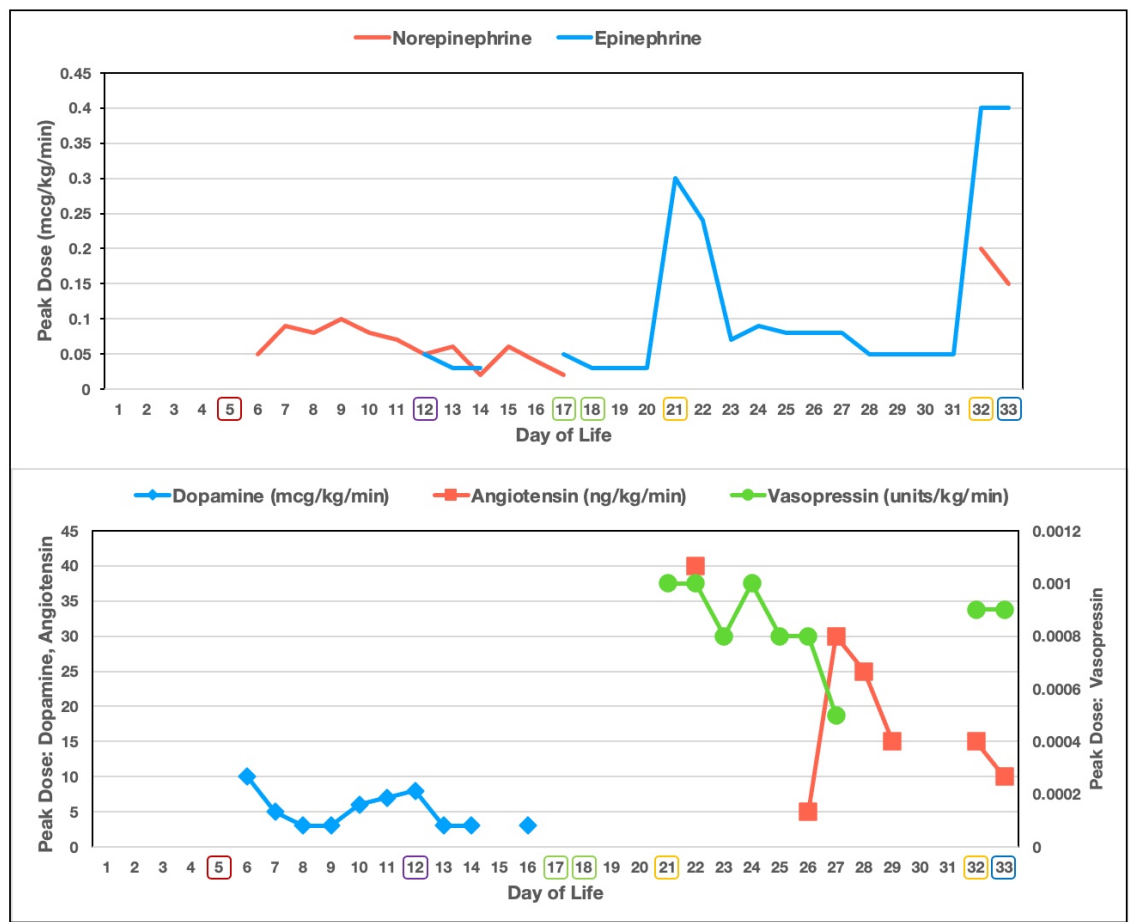

Figure 1. Vasopressor peak daily dosing. (5) Clinical sepsis found. 12] Cardiac surgery (post-operative day 0). 17,18 Leech therapy used. 21, 32 ECMO Decannulation attempt. 33 Death. Mcg: microgram. Kg: kilogram. Min: minute. Ng: nanogram. 


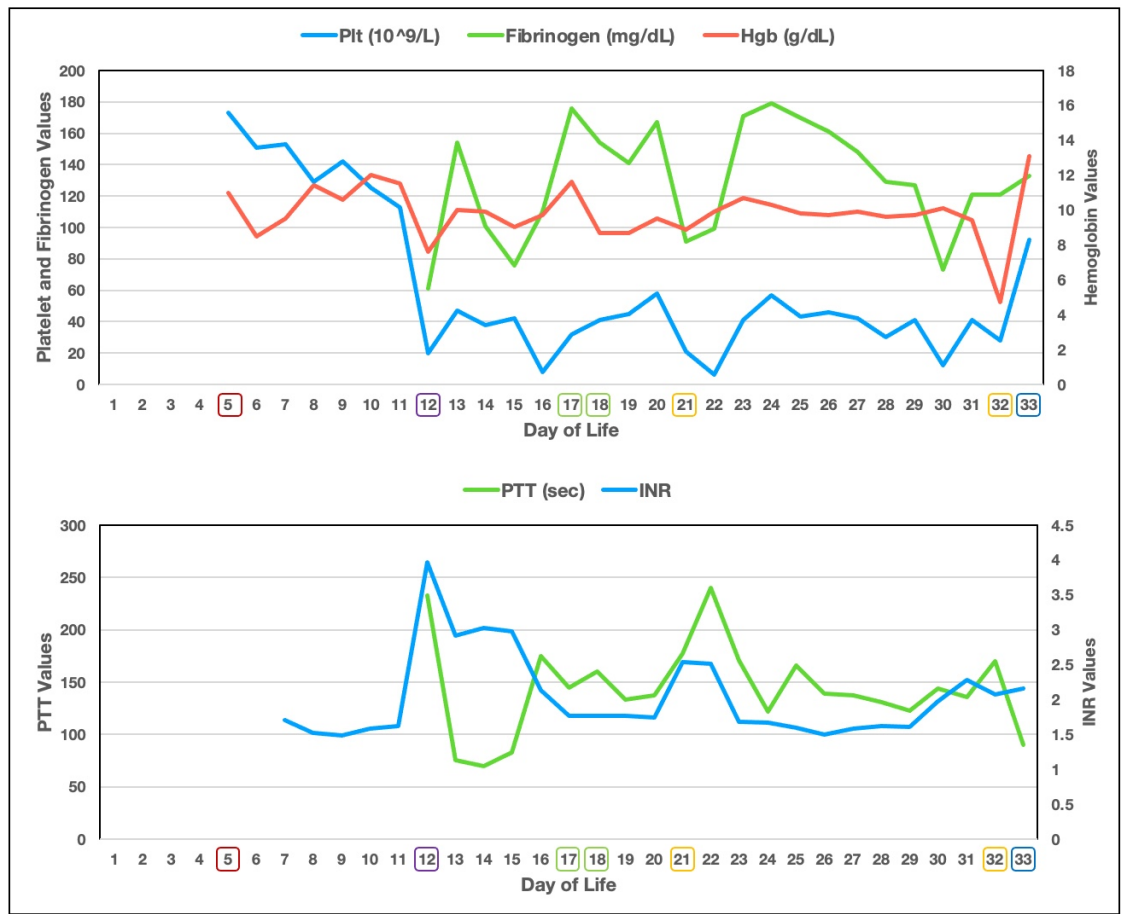

Figure 2. Daily markers of blood components and coagulation status. 5 Clinical sepsis found. 12 Cardiac surgery (post-operative day 0). 17, 18 Leech therapy used. 21, 32 ECMO Decannulation attempt. 33 Death. Plt: platelet. Hgb: hemoglobin. INR: International normalized ratio. 


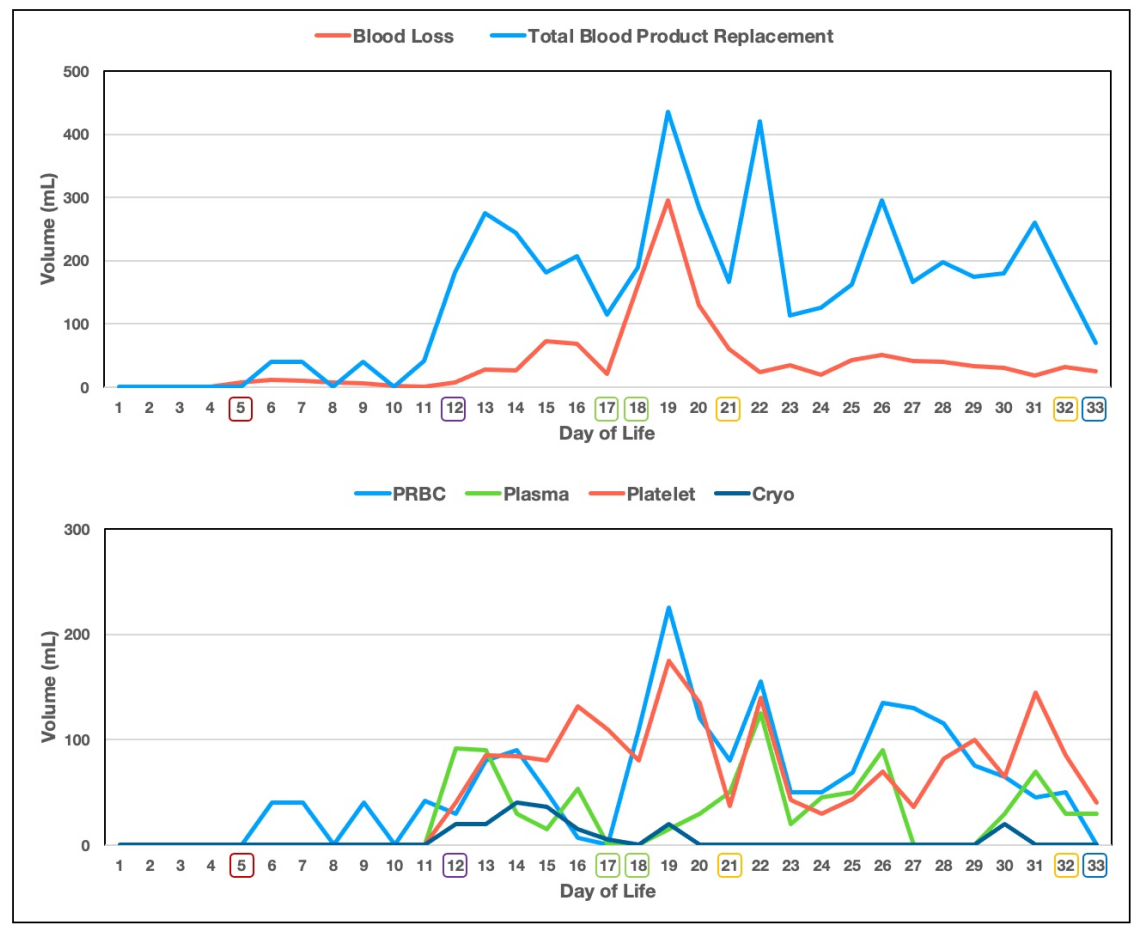

Figure 3. Blood loss, total blood product replacement, and individual product requirement on a daily basis. 5 Clinical sepsis found. 12 Cardiac surgery (post-operative day 0). 17,18 Leech therapy used. 21, 32 ECMO Decannulation attempt. 33 Death. PRBC: Packed red blood cell. Cryo: cryopreciptate.
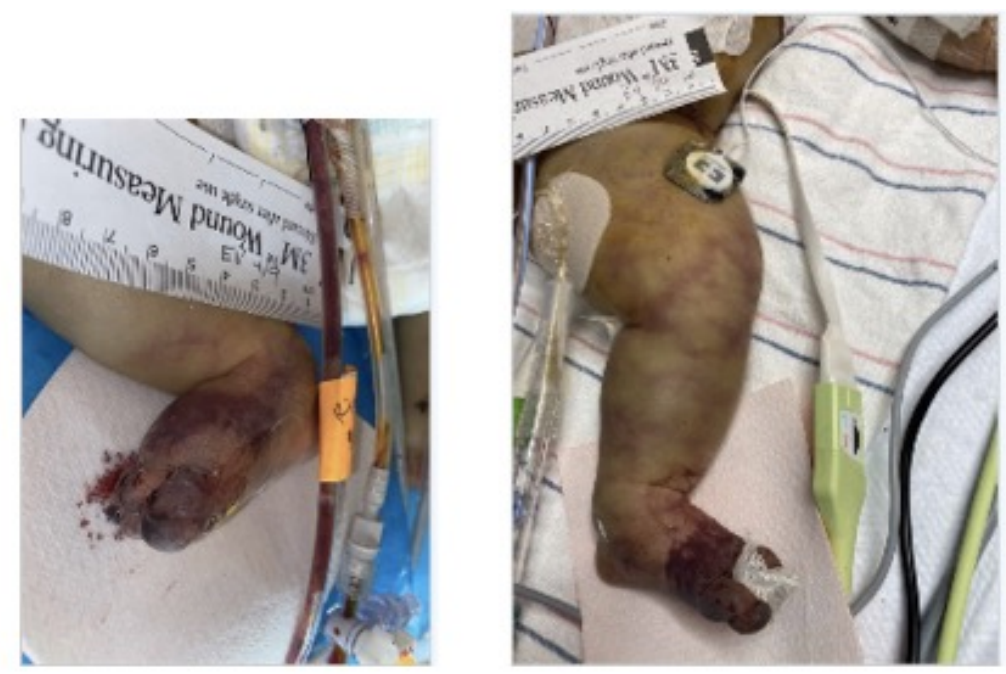

Figure 4. Visual Depiction of lower limb ischemia. Left picture: inght lower extremity on Du20 (post-operative day 8). Right picture: left lower extremity on D\#22 (post-operative day 10). 